\title{
Presentación del debate "El futuro del trabajo ante los retos de la economía de plataformas y de la industria 4.0"
}

\author{
Carlos J. Fernández Rodríguez \\ Universidad Autónoma de Madrid \\ carlos.fernandez@uam.es
}

Uno de los debates más candentes en el espacio de la sociología del trabajo y las organizaciones desde hace décadas es, sin lugar a dudas, el de los retos del empleo ante la progresiva tecnificación del aparato productivo y la proliferación de nuevas soluciones, robóticas y digitales, que a priori pueden transformar de forma radical las industrias del futuro (ver Gurrutxaga y Galarraga, 2017; Lahera Sánchez, 2019). La premonición de que la tecnología cambiará por completo el mercado de trabajo ha sido una constante, desde el clásico texto de Rifkin (2010) "El fin del trabajo", hasta las advertencias de una desaparición del empleo convencional ante la emergencia de una economía colaborativa sin intermediarios (Botsman y Rogers, 2010). La consolidación, en la pasada década, de un nuevo paradigma productivo edificado sobre los pilares de las denominadas Industria 4.0 y la economía de plataformas o gig economy parece confirmar, a priori, algunas de estas predicciones: la puesta en marcha de una nueva forma de gestión organizativa y comercial basada en la utilización de aplicaciones digitales, algoritmos, y un uso intensivo de la robótica y la inteligencia artificial podría, sin duda, tener consecuencias determinantes en el tipo y volumen de empleo disponible. Sin embargo, buena parte de las lecturas que se han hecho sobre estos impactos se han hecho desde una perspectiva demasiado influida por el determinismo tecnológico (Boyd y Holton, 2018), y merece la pena explorar desde una mirada sociológica qué es lo que está sucediendo.

La industria 4.0 se define fundamentalmente por la aplicación de tecnologías destinadas a la mayor automatización de procesos, mediante la utilización de la inteligencia artificial y la robótica, y una creciente interconectividad que permita una gestión de la información en tiempo real. El uso de nuevas herramientas como el 5G, las impresoras 3D o el recurso a las fábricas inteligentes permitiría en el futuro próximo una producción automatizada y a la vez centrada en las necesidades personales del consumidor, mientras se generan culturas de innovación en las organizaciones (Susskind y Susskind, 2015). Este nuevo paradigma industrial tendría como base el uso del denominado big data (datos acumulados gracias al internet de las cosas y a la sofisticación de la recogida de datos a través de diversos canales) y la gestión a través de algoritmos. Pero además de la avanzadilla de una Cuarta Revolución Industrial, esta industria 4.0 implica, además (y esto es lo que nos interesa), un programa social, destinado a enfrentar los futuros desafios económicos, sociales y políticos a los que se enfrentará el siglo XXI, en un esfuerzo por controlar un futuro cargado de incertidumbre (Michelsen, 2020). Por su parte, la economía de plataformas se caracteriza, precisamente, por la proliferación en el mercado de plataformas digitales, accesibles a través de internet o aplicaciones, que actúan como intermediarios entre consumidores, por un lado, y productores y oferentes de servicios por el otro. En el caso de los oferentes, generalmente trabajadores autónomos que aspiran a 
Presentación del debate "el futuro del trabajo ante los retos de la economía de plataformas y de la industria 4.0"

prestar unos servicios determinados (desde traducir un texto a entregar un paquete), estos recurren a ciertas plataformas (Upwork, Uber, Glovo) con el fin de generar ingresos contando con cierta flexibilidad, mientras los consumidores pasan a tener la posibilidad de adquirir cualquier producto o servicio con solo hacer un clic. Las plataformas, aunque se denominen colaborativas, tienen como objetivo expreso la monetización y la búsqueda de rentabilidad económica (Alonso, Fernández Rodríguez e Ibáñez Rojo, 2020). La tecnología del algoritmo, que algunos autores han bautizado como algocracia (Galiere, 2020), será como en el caso de la industria 4.0 el elemento distintivo en la gestión de estas organizaciones digitales y sus procesos de trabajo, permitiendo integrar de forma eficiente y accesible a demandantes y oferentes de distintos servicios y productos (Lehdonvirta, 2018). Tanto la industria 4.0 como la economía de plataformas conformarian la base de una nueva revolución industrial que prometería un auténtico paraíso para el consumidor: un catálogo de productos y servicios disponibles prácticamente infinito; la muerte de la distancia, con la entrega en casa; máxima rapidez en la gestión y una facilidad extraordinaria de realizar las transacciones; posibilidad de valorar el producto o servicio prestado públicamente; y una máxima personalización de la experiencia de consumo.

No obstante, las promesas de este nuevo paradigma se ven acompañadas de riesgos. Hay un temor en la sociedad a que la extrema flexibilidad que demanda este nuevo paradigma productivo contribuya a volatilizar, de forma significativa, la condición de empleo fordista (entendida como una relación laboral estable en el tiempo, con derechos y obligaciones, y con condiciones negociadas en el espacio de las relaciones industriales), e incluso generar en el futuro situaciones de desempleo masivo (Lobera, Fernández Rodríguez y Torres-Albero, 2020). En la opinión pública, hay preocupación por el impacto que las nuevas plataformas ya están teniendo en el empleo, y que se refleja en la publicación sistemática de noticias de prensa sobre la degradación del trabajo en este sector: por ejemplo, las malas condiciones laborales de los trabajadores precarios que transitan por la vía pública con bicicletas o furgonetas (los riders) repartiendo todo tipo de paquetes; los duros requerimientos y disciplina a la que se someten a los empleados del gigante Amazon; o el crecimiento de trabajos esporádicos contratados a través de aplicaciones, que termina derivando en una extraordinaria flexibilización de las relaciones laborales una vez que el trabajador va a pasar a asumir los riesgos de mercado que habitualmente formaban parte del emprendimiento empresarial, limitándose estas plataformas a gestionar los flujos comunicativos de un ágora mercantil virtual. El empleo en estos sectores ha terminado asociado a una enorme precariedad y vulnerabilidad, derivada no solo, en muchos casos, de la baja calidad del trabajo, sino de la total subordinación a una gestión algorítmica del proceso de trabajo y comercialización, la cual no solamente tiene en cuenta entregas, puntualidad y objetivos sino también sistemas de valoraciones destinados a puntuar la satisfacción por el producto y/o servicio entregado/ prestado (ver por ejemplo Revilla y Blázquez Martín, 2021). Esto obliga a una autodisciplina extraordinaria, que incentiva que los trabajadores se vean obligados a trabajar muchas horas a un ritmo infernal y estén permanentemente disponibles para poder conseguir unos ingresos suficientes y valoraciones altas (Crouch, 2019). La multiplicación, además, de un colectivo como el de estos trabajadores autónomos, cuyo estatus es de hecho bastante discutible, ha sido acogida con gran preocupación por sindicatos y expertos, lo que ha llevado a esfuerzos por parte de los gobiernos de cara a regular las "zonas grises" de la legislación laboral con el fin de zanjar las ambigüedades derivadas de estas nuevas realidades laborales (ver Serrano-Pascual y Jepsen, 2019). Finalmente, hay una preocupación en relación a otros impactos que esta nueva economía puede tener, en aspectos como el medio ambiente o el papel de las autoridades a la hora de regular estas actividades (Murillo, Buckland y Val, 2017).

Indudablemente, todas estas cuestiones relacionadas con el surgimiento de este nuevo paradigma industrial, organizativo y comercial, y su extraordinario impacto social, hacen relevante que, en la Revista Española de Sociología, se haya procedido, como en otras ocasiones con otros temas, a organizar una sección de debate destinada a discutir las implicaciones e impactos que esta nueva "revolución industrial" puede tener sobre 
las sociedades contemporáneas. Para ello, se ha procedido a contar con la colaboración de una serie de expertos que han orientado sus contribuciones a reflexionar sobre aspectos concretos derivados de la digitalización de la economía. Evidentemente, los aspectos tratados aquí no agotan la cantidad extraordinaria de temas de investigación que están en este momento floreciendo en torno al universo de la Industria 4.0 y la platform economy o gig economy, pero no cabe duda de que sin duda serán útiles para introducir al lector de la RES a algunos de los temas más candentes en este campo de investigación.

Los textos que conforman esta sección son cuatro. En el primero, el profesor Arturo Lahera de la Universidad Complutense de Madrid, uno de los más destacados sociólogos del trabajo de nuestro país, presenta una interesante revisión del "estado del arte" en torno a los debates sobre el citado desempleo tecnológico masivo futuro que están teniendo lugar en las ciencias sociales a día de hoy. Lahera discute en su texto una serie de enfoques en torno a la automatización derivada del proceso de digitalización y robotización asociado a las nuevas tecnologías de la Industria 4.0, cartografiando los discursos más relevantes en torno a estas tendencias de fondo. El autor propone un cuestionamiento crítico de los mismos, apostando por visibilizar el trabajo humano que está sustentando todos estos procesos automatizados de la producción digital. Por su parte, Gloria Álvarez y Óscar Pérez Zapata, profesores de la Carlos III y que llevan ya tiempo desarrollando interesantes investigaciones en el espacio de la sociología del trabajo - particularmente sobre el impacto del management y la organización del trabajo en la salud (ver p.e. Pérez Zapata, 2019) - aportan como contribución a este debate un interesante estudio de caso centrado en las condiciones de trabajo en una plataforma del sector de la educación online. En su análisis, Álvarez y Pérez Zapata analizan críticamente la gestión "platafórmica" que, más allá del algoritmo, genera procesos de gestión de las competencias personales que conllevan una intensificación notable del trabajo cognitivo y emocional, frente a las promesas de autonomía y flexibilidad publicitadas por la plataforma, con consecuencias en la salud de los empleados. Ciertamente, estos desarrollos en el campo de la educación son indudablemente inquietantes y anticipan una futura extensión de estas prácticas a otros espacios del trabajo cualificado.

La tercera contribución está firmada por un equipo de excelentes investigadores de la Universidad de Manchester (Miguel Martínez Lucio, Stephen Mustchin, Stefania Marino, Debra Howcroft y Hollie Smith), entre los cuales algunos lectores de la RES identificarán al profesor Martínez Lucio, una referencia para muchos académicos españoles en el terreno de las relaciones laborales y los estudios sobre sindicalismo. El texto, que publicamos en su inglés original, tiene como objetivo revisar la situación de los sindicatos ante el ascenso de la gig economy, que es evidentemente complicada una vez que no solamente se está experimentando una degradación de las condiciones de trabajo, sino que, además, la organización colectiva de los trabajadores en ese sector es complicada por el estatus difuso de los trabajadores (contratados como autónomos) y por las dinámicas individualizadoras que la condición de emprendedor generan. Los autores resaltan que, frente a las miradas más pesimistas que enfatizan la impotencia sindical en este contexto, recientes investigaciones muestran que algunos sindicatos han procedido a una estrategia de renovación destinada a defender los intereses de los trabajadores, pese a los evidentes desafíos que estas innovaciones tecnológicas puedan suponer. Finalmente, la última contribución está firmada por Luis Enrique Alonso, uno de los más importantes especialistas en sociología del consumo en España, y el autor de esta introducción, ambos profesores de la Universidad Autónoma. En este trabajo, nuestro objetivo es el de poner el énfasis en un elemento generalmente minusvalorado cuando analizamos el creciente éxito de las plataformas: la centralidad que el consumo tiene a la hora de alimentar estos modelos de negocio. No puede existir un paradigma como el de la economía de plataformas sin la aparición y consolidación de formas de consumo y estilos de vida muy concretos, vinculados a la creciente digitalización de la economía, el uso extendido de los algoritmos como nueva estrategia de identificación de los consumidores potenciales, y una cultura de hiperconsumo probablemente 
insostenible a largo plazo. En este texto, reflexionamos sobre los costes que, para el universo del trabajo, tienen las prácticas de consumo contemporáneas, haciendo una llamada para visibilizar ese vínculo, tantas veces oculto o minusvalorado, entre ambos espacios.

En resumen, las cuatro contribuciones aquí presentadas tratan cuestiones diversas, que nos muestran la compleja naturaleza de esta economía basada en la industria 4.0 y las plataformas digitales, y sus repercusiones en diversas esferas del mundo social, particularmente la del espacio del trabajo en nuestra sociedad y su futuro ante el rápido avance tecnológico. Confiamos en que este debate capte el interés de los lectores de la RES, y sirva para estimular nuevas reflexiones en nuestra comunidad sociológica en torno a los desafios futuros que supone esta transformación digital de la economía en todos los órdenes de la vida.

\section{REFERENCIAS}

Alonso, L. E., Fernández Rodríguez, C. J., Ibáñez Rojo, R. (2020). Del low cost a la gig economy: el consumo en el postfordismo del siglo XXI. En Alonso, Luis E., Fernández Rodríguez, C. J., Ibáñez Rojo, R. (Eds.), Estudios sociales sobre el consumo (pp.241-260). Madrid: Centro de Investigaciones Sociológicas.

Botsman R., Rodgers, R. (2010). What's Mine Is Yours: The Rise of Collaborative Consumption. Nueva York: Harper Collins.

Boyd R., Holton R.J. (2018). Technology, innovation, employment and power: Does robotics and artificial intelligence really mean social transformation? Journal of Sociology, 54(3): 331-345. doi:10.1177/1440783317726591

Crouch, C. (2019). Will the Gig Economy Prevail? Cambridge: Polity.

Galiere, S. (2020). When food-delivery platform workers consent to algorithmic management: a Foucauldian perspective. New Technology, Work and Employment, 35(3), 357-390. https://doi.org/10.1111/ntwe.12177

Gurrutxaga, A., Galarraga, A. (2017). Fábricas del futuro. Conocimiento social y tecnología. Madrid: Plaza y Valdés.

Lahera Sánchez, A. (2019). Digitalización, robotización, trabajo y vida: cartografías, debates y prácticas. Cuadernos De Relaciones Laborales, 37(2), 249-273.https://doi.org/10.5209/ crla.66037

Lehdonvirta, V. (2018). Flexibility in the gig economy: managing time on three online piecework platforms. New Technology, Work and Employment, 33(1): 13-29. https://doi. org/10.1111/ntwe.12102

Lobera, J., Fernández Rodríguez, C. J., Torres-Albero, C. (2020). Privacy, Values and Machines: Predicting Opposition to Artificial Intelligence. Communication Studies, 71(3), 448-465. https://doi.org/10.1080/10510974.2020.1736114

Michelsen, K.-E. (2020). Industry 4.0 in Retrospect and in Context. In Collan, M., Michelsen, K.-E. (Eds.), Technical, Economic and Societal Effects of Manufacturing 4.0. Palgrave MacMillan. https://doi.org/10.1007/978-3-030-46103-4

Murillo, D., Buckland, H., Val, E. (2017). When the sharing economy becomes neoliberalism on steroids: Unravelling the controversies. Technological Forecasting \& Social Change, 125: 66-76. https://doi.org/10.1016/j.techfore.2017.05.024 
Pérez Zapata, O. (2019). Trabajo sin límites, salud insostenible: la intensificación del trabajo del conocimiento. Madrid: Marcial Pons.

Revilla, J. C., Blázquez Martín, V. (2021). Uneasy riders: contradictorias lógicas disciplinarias para una posición laboral imposible. Revista Española de Sociología 30(2), a35. https:// doi.org/10.22325/fes/res.2021.35.

Rifkin, J. (2010). El fin del trabajo. Barcelona: Paidós.

Serrano-Pascual, A. Jepsen, M. (Eds.) (2019). The Deconstruction of Employment as a Political Question: "Employment" as a Floating Signifier. Nueva York: Palgrave.

Susskind R., Susskind, D. (2015). The Future of the Professions: How Technology will Transform the Work of Human Experts. Oxford: Oxford University Press. 